\title{
Workshop on Robots for Learning - R4L
}

\author{
Wafa Johal \\ CHILI/LSRO Labs \\ École Polytechnique \\ Fédérale Lausanne \\ Lausanne, Switzerland \\ wafa.johal@epfl.ch \\ Mirjam de Haas \\ Tilburg center for Cognition \\ and Computation \\ Tilburg University \\ Netherlands \\ mirjam.dehaas@uvt.nl
}

\author{
Paul Vogt \\ Tilburg center for Cognition \\ and Computation \\ Tilburg University \\ Netherlands \\ p.a.vogt@uvt.nl \\ Ana Paiva \\ Instituto Superior Técnico \\ University of Lisbon \\ Portugal \\ ana.paiva@inesc-id.pt
}

\author{
James Kennedy \\ Centre for Robotics and \\ Neural Systems \\ Plymouth University \\ United Kingdom \\ james.kennedy \\ @plymouth.ac.uk \\ Ginevra Castellano \\ Department of Information \\ Technology \\ Uppsala University \\ Sweden \\ ginevra.castellano \\ @it.uu.se
}

\begin{abstract}
While robots have been popular as a tool for STEM teaching, the use of robots in other learning scenarios is novel. The field of HRI has started to report on how to make effective robots usable in educational contexts. However, many challenges remain. For instance, which interaction strategies aid learning, and which hamper learning? How can we deal with the current technical limitations of robots? Answering these and other questions requires a multidisciplinary effort, including contributions from pedagogy, developmental psychology, (computational) linguistics, artificial intelligence and HRI, among others. This abstract provides a brief overview of the current state-of-the-art in social robots designed for learning and describes the aims of the Robots for Learning (R4L) workshop in bringing together a multidisciplinary audience for furthering the development of market-ready educational robots.
\end{abstract}

\section{Keywords}

Human-Robot Interaction; Robots in Education; Tutor Robots; Child-Robot Interaction

\section{INTRODUCTION \& BACKGROUND}

An increasing amount of Human-Robot Interaction (HRI) research is focused on the development of applications of service robots in everyday life. In education, while robots have been popular as a focus for STEM teaching (cf. Lego Mindstorms or Thymio [7]), the use of robots in other learning scenarios is novel.

Mubin et al. 5 distinguish three roles for robots in edu-

Permission to make digital or hard copies of part or all of this work for personal or classroom use is granted without fee provided that copies are not made or distributed for profit or commercial advantage and that copies bear this notice and the full citation on the first page. Copyrights for third-party components of this work must be honored. For all other uses, contact the owner/author(s).

HRI '17 Companion March 06-09, 2017, Vienna, Austria

(C) 2017 Copyright held by the owner/author(s).

ACM ISBN 978-1-4503-4885-0/17/03.

DOI: http://dx.doi.org/10.1145/3029798.3029801 cation: 1) tutors - providing help to students, 2) peers stimulating learning, and 3) tools - physically enhancing a concept to learn. In the 1970's and 80's robots tended to be introduced in schools as a tool for teachers to teach robotics or other STEM subjects. However, this specificity of robot usage penalized their adoption in educational contexts 2. Nowadays, with robots being cheaper and more easily deployable, application in education becomes possible for other types of learning.

The field of HRI has started reporting on how to make effective robots and how to measure their efficacy 3.8 . Robots have the potential to enhance learning via kinesthetic interaction, can improve the learner's self-esteem, and can provide empathic feedback $1,4,4,9$. Finally, robots have been shown to engage the learner, to motivate her in the learning task or to stimulate collaboration in a group 6. However, many challenges remain and this workshop aims to bring together a multidisciplinary group of researchers to discuss these challenges and share expertise. Such challenges and questions that are yet to be comprehensively addressed by the research community include: the effective involvement of education practitioners in the design of activities, the outcome of long-term learning with robots, appropriate educational strategies for use in HRI, and the influence of HRI on affective aspects of learning, such as motivation and self-efficacy.

The second iteration of this workshop builds on the previous version hosted at the IEEE International Symposium on Robot and Human Interactive Communication (RO-MAN), 2016. The previous workshop utilised keynote speakers, participant speakers, and small group discussions to raise issues and challenges facing the community researching robots for use in delivering educational content. The second version of this workshop seeks to engage with more researchers in the field, and draw a more multidisciplinary audience to further the development of market-ready educational robots.

\section{OUTLINE OF THE WORKSHOP}

The aim of this workshop is to engage scholars who wish to gain expertise in education and in robotics. Participants will benefit from hearing from the forefront of field and 
from discussions on how to move from fundamental research towards the development of market-ready educational robots.

The workshop aims will be achieved through presentations and discussions. Prospective participants are invited to submit 4-6 page papers describing work in progress, or containing preliminary results to discuss with the community. In order to stimulate interactions, the workshop will include short position paper presentations and poster sessions. The afternoon will be dedicated to discussion, including both a panel session and semi-structured group discussions.

\section{ORGANIZERS}

Wafa Johal, PhD. is a postdoctoral researcher within the CoWriter and Cellulo projects dealing with robots for education in the CHILI and LSRO Labs at EPFL. She obtained her $\mathrm{PhD}$ in 2015 from the University of Grenoble (France) focusing on body signals in Child-Robot Interaction.

Paul Vogt is Associate Professor in Language learning and HRI. He is a trained cognitive scientist and holds a PhD in Artificial Intelligence. His research focuses on 1st and 2nd language acquisition using methods from ethnographic research, psycholinguistics, computational modelling of language acquisition and HRI. Paul is one of the principal investigators in the L2TOR project.

James Kennedy is currently completing his $\mathrm{PhD}$ in HumanRobot Interaction at Plymouth University (U.K.). His research interests centre around social companion robots, particularly in educational interactions with children. He has been involved with the ALIZ-E, DREAM and L2TOR European projects.

Mirjam de Haas finished her Master's degree in Artificial Intelligence and is a $\mathrm{PhD}$ student in the L2TOR project. Her research focuses on the interaction between robots and children and how to design a child-friendly robot.

Ana Maria Paiva's main scientific interests lie in the area of Autonomous Agents, Embodied Conversational Agents and Robots, and Multiagent Simulation Systems. She has been researching in the area of artificial intelligence for the past twenty years. She is the principal investigator of the eCUTE project aiming to explore technologically-enhanced learning approaches for inter-cultural understanding.

Ginevra Castellano is an associate senior lecturer in intelligent interactive systems at Uppsala University, where she leads the Social Robotics Lab. She was the coordinator of the EMOTE project, which developed educational robots to support teachers in a classroom environment.

Sandra Okita is an Associate Professor of Technology and Education at Teachers College, Columbia University. Her current research interest is focused on the learning partnership between individuals and technology, and how technology intersects with learning and instructional processes.

Fumihide Tanaka, $\mathrm{PhD}$, has been actively working in the area of educational robots and child-robot interaction, and is now recognized as one of the pioneers in this research area. He moved to academia in 2008, the University of Tokyo (2014), and is currently at the University of Tsukuba, Japan.

Tony Belpaeme's research focuses on cognitive robotics and social Human-Robot Interaction, in which natural and artificial cognition is considered to be closely intertwined with social interaction. He coordinates the L2TOR project on learning a 2nd language using robot tutors, and collaborates on several international research projects on HRI and cognitive robotics.
Pierre Dillenbourg is a former elementary school teacher. He graduated in educational science (University of Mons, Belgium). His research on learning technologies started in 1984 He obtained a $\mathrm{PhD}$ in computer science from the University of Lancaster (UK), in artificial intelligence applications for educational software. He is currently full professor in learning technologies, head of the CHILI Lab involved in both CoWriter and Cellulo projects.

\section{ACKNOWLEDGMENTS}

We would like to thank the Swiss National Science Foundation National Centre of Competence in Research Robotics and the EU H2020 L2TOR project (grant no. 688014).

\section{ADDITIONAL AUTHORS}

- Sandra Okita, Teachers College - Columbia University, United States, okita@tc.columbia.edu

- Fumihide Tanaka, University of Tsukuba, Japan, tanaka@iit.tsukuba.ac.jp

- Tony Belpaeme, Centre for Robotics and Neural Systems, Plymouth University, U.K. and Ghent University, Belgium, tony.belpaeme@plymouth.ac.uk

- Pierre Dillenbourg, CHILI Lab, École Fédérale Polytechnique Lausanne, Switzerland, pierre.dillenbourg@epfl.ch

\section{REFERENCES}

[1] G. Castellano, A. Paiva, A. Kappas, R. Aylett, H. Hastie, W. Barendregt, F. Nabais, and S. Bull. Towards empathic virtual and robotic tutors. In Artificial Intelligence in Education, pages 733-736. Springer, 2013.

[2] W. Gander, A. Petit, G. Berry, B. Demo, J. Vahrenhold, A. McGettrick, R. Boyle, M. Drechsler, A. Mendelson, C. Stephenson, C. Ghezzi, and B. Meyer. Informatics education: Europe cannot afford to miss the boat, Report of the joint Informatics Europe \& ACM Europe Working Group on Informatics Education. Available at: http://europe.acm.org/iereport/ie.html, 2013.

[3] J. Kennedy, P. Baxter, E. Senft, and T. Belpaeme. Social Robot Tutoring for Child Second Language Learning. In Proceedings of the 11th ACM/IEEE International Conference on Human-Robot Interaction, pages 67-74. ACM, 2016.

[4] S. Lemaignan et al. Learning by Teaching a Robot: The Case of Handwriting IEEE Robotics Automation Magazine, 23(2):56-66, 2016.

[5] O. Mubin, C. J. Stevens, S. Shahid, A. A. Mahmud, and J.-J. Dong. A review of the applicability of robots in education Journal of Technology in Education and Learning, 1:209-0015, 2013.

[6] H. W. Park and A. Howard. Providing tablets as collaborative-task workspace for human-robot interaction. In Proceedings of the 8th ACM/IEEE international conference on Human-robot interaction, pages 207-208. IEEE Press, 2013.

[7] F. Riedo, P. Rétornaz, L. Bergeron, N. Nyffeler, and F. Mondada. A Two Years Informal Learning Experience Using the Thymio Robot In Advances in Autonomous Mini Robots, pages 37-48. Springer, 2012.

[8] F. Tanaka, K. Isshiki, F. Takahashi, M. Uekusa, R. Sei, and K. Hayashi. Pepper learns together with children: Development of an educational application. In IEEE-RAS 15th International Conference on Humanoid Robots, HUMANOIDS 2015, pages 270-275. IEEE, 2015.

[9] F. Tanaka and S. Matsuzoe. Children teach a care-receiving robot to promote their learning: Field experiments in a classroom for vocabulary learning. Journal of Human-Robot Interaction, 1(1):78-95, 2012. 